Available online at GSC Online Press Directory

GSC Biological and Pharmaceutical Sciences

e-ISSN: 2581-3250, CODEN (USA): GBPSC2

Journal homepage: https://www.gsconlinepress.com/journals/gscbps

(RESEARCH ARTICLE)

\title{
Assessment of lipid peroxidation, antioxidant enzyme superoxide dismutase, glutathione and serum homocysteine level in patients with polycystic ovary syndrome in Sudan Khartoum state
}

\author{
Ahmed Nassir Mohamed ${ }^{1}$, Noon Babiker Mohammed ${ }^{2,}{ }^{*}$, Abdelgadir Elamin Eltom ${ }^{3}$ and Amin Omer Abbas ${ }^{4}$ \\ ${ }^{1}$ Department of Clinical Chemistry, Alfalah Medical Polyclinic,Taif, Saudi Arabia. \\ ${ }^{2}$ Associate professor in clinical chemistry University of Science and Technology, Sudan. \\ ${ }^{3}$ Medical Laboratories Sciences, College of Health Sciences, Gulf Medical University, Ajman, UAE. \\ 4 Department of Immunohematology, Albakkari Polyclinic, Almadinah Almunawarah, Saudi Arabia.
}

Publication history: Received on 17 September 2020; revised on 27 September 2020; accepted on 28 September 2020

Article DOI: https://doi.org/10.30574/gscbps.2020.12.3.0300

\begin{abstract}
Polycystic ovary syndrome (PCOS) is a common health problem that affects teenage girls and young women. PCOS is associated with a wide spectrum of complications in various parts of health, including reproductive, metabolic and psychological features. Analytical case control study was conducted to Assessment of Lipid peroxidation, antioxidant enzyme Super oxide dismutase, Glutathione and Serum Homocysteine level in Patients with (PCOS) in Khartoum State. A total of three hundred individuals were enrolled in this study, and classified into two groups, two hundred patients with (PCOS) as test group and hundred healthy individuals as control group, the samples were used to measure Homocysteine, Glutathione, Lipid peroxidation and superoxide dismutase level in the serum of participant with increased body mass index in study group and normal body mass index. The results were expressed as (mean \pm SD).The mean \pm standard deviation of the total glutathione was found mean $1174.5 \pm 271.5$ and the reduced glutathione was $(801.30 \pm 132.2)$, and for superoxide dismutase mean \pm standard deviation $(225.2, \pm 57.8)$ in study group while in control group found 195.5, \pm 25.6 respectively, Homocysteine mean \pm standard deviation $(14.9, \pm 2.1)$ for test group and $(12.1, \pm 2.5)$ for control group. Lipid peroxidation mean \pm standard deviation was found $3.4 \pm 1.1$ for test group and control group $(2.4 \pm 0.7)$ respectively. The present study has conculcated that mean of serum homocysteine, glutathione, lipid peroxide and superoxidase concentrations were increased in women with (PCOS), could be a dilemma for many women of reproductive ages irrespective of their reproductive disorders.
\end{abstract}

Keywords: Polycystic ovary syndrome (PCOS); Lipid peroxidation; Antioxidant enzyme Superoxide dismutase;

\section{Introduction}

Polycystic ovary syndrome (PCOS) is a major commonly occurring endocrine disorder with prevalence of $5 \%-21 \%$ among reproductive-aged women. PCOS is associated with a wide spectrum of complications in various parts of health, including reproductive, metabolic and psychological features. Several mechanisms suggested to contribute to the pathogenesis of PCOS include hormonal imbalance, resistance to insulin and genetic inheritance (1). The primary cause of PCOS is multifactorial in origin, though hyperandrogenism, polycystic ovaries and oligo-anovulation are common features in women with PCOS (2).

\footnotetext{
${ }^{*}$ Corresponding author: Noon Babiker Mohammed

Associate professor in clinical chemistry University of Science and Technology, Sudan. 
Hyperandrogenism favors excess luteinizing hormone secretion in fetal life in hypothalamic-pituitary unit programming, bringing about the development of insulin resistance and abdominal obesity (3). Also, disruption of ovulatory function (anovulation) in PCOS may result from both altered steroid negative feedback regulation of luteinizing hormone and the compensatory hyperinsulinemia caused by insulin resistance (4). The Rotterdam Consensus Criteria established by the European Society for Human Reproduction and Embryology and American Society for Reproductive Medicine in 2003 dependent on the broad studies during the most recent decades, have been used as diagnosing criteria for PCOS (5) . However, lifestyle modification, meditation, weight loss, herbal medicines have been used in the management of PCOS (1).

PCOS is a heterogeneous disorder that affects at least 7\% of adult women (6). According to the National Institutes of Health Office of Disease Prevention, PCOS affects approximately 5 million women of childbearing age in the U.S. Costs to the U.S. health care system for the identification and management of PCOS are approximately $\$ 4$ billion per year (7).

Research suggests that $5 \%$ to $10 \%$ of females 18 to 44 years of age are affected by PCOS, making it the most common endocrine abnormality among women of reproductive age in the U.S (8). Women seeking help from health care professionals to resolve issues of obesity, acne, amenorrhea, excessive hair growth, and infertility often receive a diagnosis of PCOS.

Women with PCOS have higher rates of endometrial cancer, cardiovascular disease, dyslipidemia, and type-2 diabetes mellitus (9).

\subsection{Objective}

\subsubsection{General objective}

To Assess of Lipid peroxidation, antioxidant enzyme Super oxide dismutase, Glutathione and Serum Homocysteine level in Patients with Polycystic Ovary Syndrome in Khartoum State.

\subsubsection{Specific objective}

To measure Lipid peroxidation, antioxidant enzyme Super oxide dismutase, Glutathione and Serum Homocysteine level in Patients with Polycystic Ovary Syndrome comparing with mean of control group.

\section{Material and methods}

\subsection{Study design}

Analytical case control.

\subsection{Study area}

The Study done in Khartoum State in Adam and Hawa Infertility Center.

\subsection{Study period}

This study carried during the period Between December 2015 up to 2019

\subsection{Study population}

The study conducted on healthy women volunteers (age: 18 - 46 years) patients with clinically proven polycystic ovary syndrome.

\subsection{Inclusion criteria}

Healthy women volunteers Patients with Poly Cystic Ovary Syndrome.

\subsection{Control group}

Normal healthy women volunteers n (100) with normal cycles between 18 - 46 years old, acted as a control group. 


\subsection{Study group}

Volunteers women (age: 18 - 46 years) patients with clinically proven polycystic ovary syndrome n (200) were chosen for the study as study subjects (patients).

\subsection{Exclusion criteria}

Hypertension, diabetic Patients, cancer, ischemic heart disease, renal disease, autoimmune disease, thyroid disease Subjects with history of receiving anti-inflammatory drugs in last 6 months and history or present symptoms of any other stress induced disorder. Hyperprolactinemia, as well as thyroid disease, were excluded.

\subsection{Sample size}

100 normal healthy women volunteers with normal cycles between $18-46$ years old, acted as a control group. And 200 women volunteers (age: 18 - 46 years) patients with clinically proven polycystic ovary syndrome were chosen for the study as study subjects (patients).

\subsection{Ethical consideration}

- The objective of the study is explained to all individuals participating in this study.

- Informed consent will obtain from the entire participant in the study.

- Health education provided to all participants.

\subsection{Data collection and questionnaire}

All the data of this study were obtained from data record. A questionnaire is specific designed to obtain information which helps in either including or excluding certain individual in the study.

\subsection{Sampling collection}

Blood samples $(7 \mathrm{ml})$ were collected from fasting subjects of the study group after fulfillment of the questionnaire as well as the control group, using disposable syringe and antiseptic alcohol [70\%ethanol] swab for skin. The samples were collected in lithium heparin containers and plain containers. Blood in heparinized containers were gently mixed with the anticoagulant to obtain plasma. And serum obtained after centrifuged plain containers at $10000 \mathrm{rpm}$ for 10 minutes at $-4^{\circ} \mathrm{C}$ using cold centrifuge.

Hemolyzed and lipemic samples were rejected and excluded from the study. The whole blood used immediately after collection for testing Glutathione. Plasma samples were preserved at - " $20{ }^{\circ} \mathrm{C}$ " prior to processing, heparinized plasma for testing Superoxide dismutase while serum sample for testing Homocysteine and Lipid peroxidation (malondialdehyde).

\subsection{Biochemical measurement and Methodologies}

\subsubsection{Materials required}

Syringe seals, plain containers, lithium heparin containers, alcohol swab, cotton and marker pens, centrifuge, cleaning solution system.

\subsubsection{Measurement of Homocysteine and Principle of the Assay}

Bound or dimerized homocysteine (oxidized form) is reduced to free homocysteine, which then reacts with serine catalyzed by cystathionine beta-synthase (CBS) to form cystathionine. Cystathionine in turn is broken down by cystathionine beta-lyase (CBL) Tao form homocysteine, pyruvate and ammonia. Pyruvate is then converted by lactate dehydrogenase (LDH) to lactate with nicotinamide adenine dinucleotide (NADH) as coenzyme. The rate of NADH conversion to NAD+ is directly proportional to the concentration of homocysteine (A340 nm).

\subsubsection{Measurement of Glutathione and Principle of the Assay}

Glutathione peroxidase (GPx) is an enzyme found in cytoplasmic and mitochondrial fractions of cells. GPx catalyses the reduction of hydrogen-peroxide and hydroperoxides formed from fatty acids, thus effective lyremoving toxic peroxides from living cells. It plays the important role of protecting cells from potential damage from free radicals formed by 
peroxide decomposition. Fortress kit is used for the quantitative determination of total Glutathione peroxidase (GPX). GPX catalyses the oxidation of Glutathione (GSH) by cumene hydroperoxide. The oxidised glutathione is converted to the reduced form in the presence of glutathione reductase and NADPH. In this reaction the NADPH is oxidized to NADP+ Simultaneously. The decrease in absorbance at $340 \mathrm{~nm}$ is then measured.

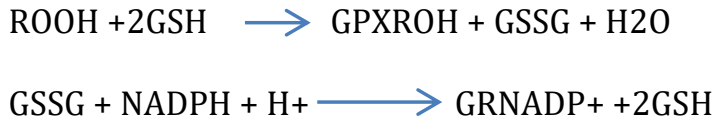

\subsubsection{Measurement of SUPEROXIDE DISMUTASE and Principle of the Assay}

Test Superoxide dismutase (SOD) role is to accelerate the dismutation of the toxic superoxide radical (02-), produced during oxidative energy processes, to hydrogen peroxide and molecular oxygen. Fortress method employs xanthine and xanthine oxidase (XOD) to generate superoxide radicals which react with 2-(4-iodophenyl)-3-(4-nitrophenol)-5phenyltetrazolium chloride (I.N.T.) to form a red form azandye. The superoxide dismutase activity is then measured by the degree of inhibition of this reaction. One unit of SOD is that which causes a $50 \%$ inhibition of the rate of reduction of INT under the conditions of the assay.

The second step is a $\beta$-elimination reaction which takes place under alkaline conditions. This reaction is mediated by reagent $\mathrm{R} 2(30 \% \mathrm{NaOH})$ which specifically transforms the substitution product (thioether) obtained with GSH into a chromophoricthione which has a maximal absorbance wavelength at $400 \mathrm{~nm}$. This method makes it possible to specifically assay glutathione with only one sampling and one colorimetric measurement. A modification of this method can be used to assay other mercaptans. This is based on the measurement of substitution products, thioethers, which absorb light at $356 \mathrm{~nm}$ in the absence of reagent R2. Because of its simplicity, this method is especially well adapted to the assay of glutathione in large series of biological samples. The main advantage of the method is the specificity for glutathione and it does not require an enzyme as a reagent.

\subsubsection{Measurement of Lipid peroxidation (malondialdehyde) and Principle of the Assay}

This assay is based on the reaction of a chromogenic reagent, 2-thiobarbituric acid, with MDA at $25^{\circ} \mathrm{C}$. One molecule of MDA reacts with 2 molecules of 2-thiobarbituric acid via a Knoevenagel-type condensation to yield a chromophore with absorbance maximum at $532 \mathrm{~nm}$,

\subsection{The body mass index (BMI)}

The body mass index (BMI) or Quetelet index is a value derived from the mass (weight) and height of an individual. The BMI is defined as the body mass divided by the square of the body height and is universally expressed in units of $\mathrm{kg} / \mathrm{m} 2$, resulting from mass in kilograms and height in meters.

The BMI is an attempt to quantify the amount of tissue mass (muscle, fat, and bone) in an individual, and then categorize that person as underweight, normal weight, overweight, or obese based on that value. However, there is some debate about where on the BMI scale the dividing lines between categories should be placed (10). Commonly accepted BMI ranges are underweight: under 18.5, normal weight: 18.5 to 25 , overweight: 25 to 30, obese: over 30.

\subsection{Interview and questionnaire}

The complete clinical and personal history of the subjects will be recorded.

\subsection{Quality control}

The precision and accuracy of all methods use in this study were checked each time a batch analyzed by including commercially prepared control sera.

\subsection{Data analysis}

The data was analyzed using statistical package of social science (SPSS) computer program using, independent t-test, results was expressed as (mean $\pm \mathrm{SD}$ ), and significance difference was considered as ( $\mathrm{P}$-value $<0.05$ ). 


\section{Results}

Patient samples were investigated to detect the mean and standard deviation to glutathione, superoxide dismutase, lipid peroxidation and serum hemoctoseine, glutathione was identify in two form total and reduced one .out of all 300 sample, were divided into 200 case and 100 control, the mean and standard deviation was found as follow the total glutathione mean 801.30 and the reduced glutathione 132.2 while in control 748.6,103.2 respectively . The reduced glutathione means \pm standard deviation was found $1174.5, \pm 271.5$ in case and 986.1, \pm 191.5 in control. When we look to superoxide dismutase the mean \pm standard deviation was found $225.2, \pm 57.8$ in study group while in control found $195.5, \pm 25.6$ respectively. Hemoctoseine mean \pm standard deviation found $14.9, \pm 2.1$ for case and $12.1, \pm 2.5$ for control lastly also the mean and standard deviation investigated for lipid peroxidation and found $3.4, \pm 1.1$ for case and $2.4, \pm$ 0.7 Respectively, In this study we found that the level of all parameters in study group was significantly increase when compared to healthy individuals. (P. value 0.00)( Table 1).

Table 1 Comparison of the means of Blood Parameters between test group and control group.

\begin{tabular}{|l|l|l|l|l|}
\hline Parameters & Mean & Std. Deviation & \multirow{2}{*}{ p.value } \\
\hline \multirow{2}{*}{ Reduce glutathione } & Case & 801.3 & 132.2 & \multirow{2}{*}{0.00} \\
\cline { 2 - 4 } & Control & 748.6 & 103.2 & \multirow{2}{*}{0.00} \\
\hline \multirow{2}{*}{ Total glutathione } & Case & 1174.5 & 271.5 & \multirow{2}{*}{0.00} \\
\cline { 2 - 5 } superoxide dismutase & Control & 986.1 & 191.5 & \multirow{2}{*}{0.00} \\
\hline \multirow{3}{*}{ Homocysteine } & Case & 225.2 & 57.8 & \multirow{2}{*}{0.00} \\
\cline { 2 - 5 } & Control & 195.5 & 25.6 & \\
\hline \multirow{2}{*}{ lipid peroxidation } & Case & 14.9 & 2.1 & 2.5 \\
\cline { 2 - 5 } & Control & 12.1 & 1.1 & \\
\hline
\end{tabular}

\section{Discussion}

Polycystic ovarian syndrome (PCOS) is one of the most common endocrine abnormalities among women in reproductive ages.

In the present study the lipid peroxidation product levels have been increased significantly in the patients with polycystic ovary syndrome compared to controls. In our study the antioxidant enzymes i.e. SOD \& GPx activities have been increased significantly in patients with PCOS compared to controls. SOD is the important antioxidant enzyme having an antitoxic effect against super oxide anion. The over expression of SOD might be an adaptive response and it results in increased dismutation of superoxide to hydrogen peroxide. GPX, an oxidative stress inducible enzyme plays a significant role in the peroxyl scavenging mechanism and in maintaining functional integration of the cell membranes (11). The rise could be due to its induction to counter the effect of increased oxidative stress.

After adjusting for confounding factors such as age, BMI, gravidity, serum vitamin E, and serum lipid levels(12) Increased lipid peroxidation have been reported in the endometrium of women with PCOS $(13,14)$ However, several studies failed to find significant differences in the , lipid peroxide, and PCOS in women.

The Glutathione is a group of multifunctional proteins, which play a central role in detoxification of electrophilic chemicals \& the hepatic removal of potentially harmful hydrophobic compounds from blood (15). We have observed a significant increase in the GS activity in patients with polycystic ovary syndrome compared to controls in which out of 300 sample divided into 200 case and 100 control, the mean and standard deviation was found as follow the total glutathione mean 801.30 and the reduced glutathione 132.2 while in control 748.6,103.2 respectively. The rise in the activity of GS could be due to its induction to counter the effect against increased oxidative stress. 
Homocysteine has been recognized recently as a risk factor for vascular diseases. In our study, Serum. Our data showed that serum homocysteine levels were significantly higher in PCOS women than controls. Our findings are consistent with a previous study by Loverro and his group (16) and Badawy et al., (17). Mancini et al., (18) in their study found no significant difference in homocysteine levels among PCOS women and controls.

Difference Studies have shown correlation between homocysteine and BMI (1) Schachter (19), he shown correlation between homocysteine and insulin resistance. Studies found that increased homocysteine levels and decreased antioxidant capacity may contribute to the increased risk of cardiovascular disease in women with PCOS (20) other study have evaluated homocysteine levels in PCOS subjects based on BMI matched subjects. In our study homocysteine levels were compared among PCOS cases and controls as well as in subgroups based on BMI and waist circumference $(21,22)$. An increased serum homocysteine level is associated with the formation of atherosclerotic plaques and myocardial infarction. Similar reports of increased levels of homocysteine in polycystic ovary syndrome were reported (23).

\section{Conclusion}

The present study has demonstrated that mean serum homocysteine, glutathione, lipid peroxide and superoxidase concentrations were increased in women with PCOS. Oxidative stress is increased in patients with polycystic ovary syndrome. The increased activities of antioxidant enzymes may be a compensatory regulation in response to increased oxidative stress. Increased homocysteine levels and decreased antioxidant capacity may contribute to the increased risk of cardiovascular disease in women with PCOS.

\section{Compliance with ethical standards}

\section{Acknowledgments}

Grateful thank to the patients and healthy who agreed to participate in this study.

\section{Disclosure of conflict of interest}

There was no conflict of interest in this study. Statement of informed consent Informed consent was obtained from all individual participants included in the study.

\section{Statement of informed consent}

Informed consent was obtained from all individual participants included in the study.

\section{References}

[1] Leila A, Najmeh T, Mansoureh M, Fahimeh RT, Saeedeh Z. Antioxidants and management of polycystic ovary syndrome in Iran: A systematic review of clinical trials. Iran J Reprod Med. 2015; 13(1): 1-8.

[2] Norman RJ, Dewailly D, Legro RS, Hickey TE. Polycystic ovary syndrome. Lancet. 2007; 370(9588): 685-697.

[3] Franks S, Berga S. Does PCOS have developmental origins? FertilSteril. 2012; 97: 2-6.

[4] Abbott DH, Barnett DK, Bruns CM, Dumesic DA. Androgen excess fetal programming of female reproduction: A developmental aetiology for polycystic ovary syndrome? Hum Reprod Update. 2005; 11: 357-374.

[5] Joham AE, Teede HJ, Ranasinha S, Zoungas S, Boyle J. Prevalence of infertility and use of fertility treatment in women with polycystic ovary syndrome: Data from a large community-based cohort study. J Wom Health. 2015; 24(4): 299-307.

[6] Aubuchon M, Legro RS. Polycystic ovary syndrome: Current infertility management. ClinObstetGynecol. 2011; 54(4): 675-684.

[7] American Congress of Obstetricians and Gynecologists. ACOG Practice Bulletin No. 108: Polycystic Ovary Syndrome. ObstetGynecol. 2009; 114(4): 936-949.

[8] National Institutes of Health, Department of Health and Human Services. Beyond Infertility: Polycystic Ovary Syndrome (PCOS). NIH Pub. No. 08-5863, April 2008. 
[9] McFarland C. Treating polycystic ovary syndrome and infertility MCN Am J Matern Child Nurs. 2012; 37(2): 116121.

[10] Dewailly D, Lujan ME, Carmina E, Cedars MI, Laven J, Norman RJ, EscobarMorreale HF. Definition and significance of polycystic ovarianmorphology: a task force from the Androgen Excess and PolycysticOvary Syndrome Society. Human Reproduction Update. 2014; 20334-352.

[11] Jenkins C, Wilson R, Roberts J, Miller H, McKillop J, Walker J. Antioxidants: Their Role in Pregnancy and Miscarriage. AntioxidRedox Signal. 2000; 2: 623-7.

[12] Szczepanska M, Kozlik J, Skrzypczak J, Mikolajczyk M. Oxidativestress may be a piece in the endometriosis puzzle. FertilSteril. 2003; 79: 1288-93.

[13] Gupta S, Agarwal A, Krajcir N, Alvarez JG. Role of oxidativestress in endometriosis. Reprod Biomed Online. 2006; 13(1): 12634 .

[14] Smith GJ, Ohl VS, Litwack G. Ligandin. The Glutathione S - Transferases, and chemically induced hepato carcinogenesis, A review, Cancer Research. 1977; 37: 8-14.

[15] Loverro G, I orussoF, Mei L, Depalo R, Cormio G, Selvaggi L. The plasma homocysteine levels are increased in polycystic ovary syndrome. Gynecolobstet Invest. 2002; 53: 157-62.

[16] Ahmed Badawy, Omnia State, Soma ShAbdElGawad and Omar Abd El Aziz. Plasmahomocysteine and polycystic ovary syndrome: themissed link. European Journal of Obstetrics and Gynaecology and Reproductive Biology. 2007; 131(1): 68- 72.

[17] Mancini F, Cianciosi A, Reggiani GM, Facchinetti F, Battaglia C, de Aloysio D. Endothelial function and its relationship to leptin, homocysteine, and insulin resistance in lean and overweight eumenorrheic women and PCOS patients: a pilot study. FertilSteril. 2009; 91(6): 2537-44.

[18] Lhan Tarkun, Berrin Çetinarslan, Zeynep Cantürk, Erdem Türemen. The Plasma Homocysteine Concentrations and Relationship with Insulin Resistance in Young Women with Polycystic Ovary Syndrome. Turkish Journal of Endocrinology and Metabolism. 2005; 1: 23-28.

[19] Mohan SK, Priya VV. Lipid peroxidation, glutathione, ascorbic acid, vitamin E, antioxidant enzyme and serum homocysteine status in patients with polycystic ovary syndrome Biology and Medicine. 2009; 1(3): 44-49.

[20] SagharSalehpour, Ozra Manzor-al-ajdad, Elham Neisani Samani, Alireza Abadi. Evaluation of Homocysteine Levels in Patients with Polycystic Ovarian Syndrome. Roya Institute. International Journal of Fertility and Sterility. 2011; 4(4): 168-71.

[21] Schachter M, Raziel A, Friedler S, Strassburger D, Bern O, Ron-El R. Insulin resistance in patients with polycystic ovary syndrome is associated with elevated plasma homocysteine. Hum Reprod. 2003; 18: 721-27.

[22] ALTOUM AEA, OSMAN AL, BABKER AM. Comparative study of levels of selective oxidative stress markers (malondialdehyde, zinc, and antioxidant vitamins A, E, and C) in ischemic and non-ischemic heart disease patients suffering from type-2 diabetes. Asian J Pharm Clin Res. 2018; 11(8): 508-510.

[23] Ahmed Badawy, Omnia State, Soma Sh.Abd El Gawad, Omar Abd El Aziz. Plasma homocysteine and polycystic ovary syndrome: the missed link, European Journal of Obstetrics and Gynaecology and Reproductive Biology. 2007; 131(1): 68 - 72 .

\section{Author's short biography}

Ahmed Nassir Mohamed
M.Sc of medical laboratory science department of chemical pathology (Alzaeim Alazhari
university) 19/06/2014.




Noon Babiker Mohammed Ahmed
Qualifications: 2014 Ph.D. in Clinical chemistry, Sudan University. Thesis entitled: Comparative
Assessment Of plasma Cystatin C, $\beta 2$ Microgloulin and Creatinine as Markers of Renal Function
in Sudanese patients with Type2 Diabetes. 2004 M.Sc in Clinical chemistry, Alzaeim Alazhare
University 1999 B.Sc. in Clinical chemistry, Faculty of Medical Laboratory Science, Ahlia
University

